\title{
Darboux Covariant Equations of von Neumann Type and their Generalizations
}

\author{
Jan L. Cieśliński ${ }^{1}$, Marek Czachor ${ }^{2,3}$ and Nikolai V. Ustinov ${ }^{4,2}$ \\ ${ }^{1}$ Uniwersytet w Białymstoku, Instytut Fizyki Teoretycznej \\ ul. Lipowa 41, 15-424 Białystok, Poland \\ ${ }^{2}$ Katedra Fizyki Teoretycznej i Metod Matematycznych \\ Politechnika Gdańska, ul. Narutowicza 11/12, 80-952 Gdańsk, Poland \\ 3 Department of Physics \\ Technische Universität Clausthal, 38678 Clausthal-Zellerfeld, Germany \\ 4 Theoretical Physics Department, Kaliningrad State University, \\ Al. Nevsky street 14, 236041, Kaliningrad, Russia
}

\begin{abstract}
Lax pairs with operator valued coefficients, which are explicitly connected by means of an additional condition, are considered. This condition is proved to be covariant with respect to the Darboux transformation of a general form. Nonlinear equations arising from the compatibility condition of the Lax pairs in the matrix case include, in particular, Nahm equations, Volterra, Bogoyavlenskii and Toda lattices. The examples of another one-, two- and multi-field lattice equations are also presented.
\end{abstract}

PACS 02.30.Ik, 05.45.Yv 


\section{Introduction}

In the present paper we consider a large class of the integrable systems of nonlinear equations taking values on an associative ring of noncommutative operators. They are defined as the compatibility condition of Lax pairs characterized by the property that the equation for the time evolution of the wave function is explicitly determined (in a local way) by the coefficients of the spectral problem. There exist different types of Lax pairs, whose coefficients are connected explicitly. Lax pairs with coefficients defined on an associative algebra of scalar pseudo-differential operators were introduced in [1, 2] (see also [3] and references therein). These Lax pairs play an important role in the Sato theory [4] and in constructing the modifications of KP hierarchy [5, 6]. The case of the shift operators and associated lattice equations were discussed in [7]. The approach, which is applied below to connect the coefficients of the Lax pairs, differs from known ones. The hierarchy of Darboux covariant nonlinear "multi-field" equations we describe in this paper contains, as the simplest case, the "one-field" equations of von Neumann type

$$
i \dot{\rho}=[H(\rho), \rho], \quad i \dot{\rho}=[H, f(\rho)] .
$$

These equations and their solutions were investigated in the context of the density matrices and Hamiltonians in [8, 9, 10], where, for instance, the formulas of the Darboux transformation were constructed. In the matrix case, the multi-field equations we derive here admit the reductions leading to known and new integrable nonlinear lattice systems.

The technique exploited in this paper combines and develops the approaches of works [11, 12]. In Sec.II we show that the relations between the coefficients of the equations forming the Lax pair, i.e. necessary conditions for the Lax pair compatibility, are identically satisfied if an additional condition on the coefficients is imposed. This condition connects explicitly the coefficients of the equations of the Lax pair and allows us to write in a closed form the nonlinear equations, which follow the compatibility condition. A theorem establishing the Darboux covariance of the Lax pair with the additional condition and, consequently, of the corresponding nonlinear equations is proved in Sec.III. This theorem gives an effective tool of producing the infinite hierarchies of solutions, including the multi-soliton solutions, for nonlinear equations and their reductions. The equations of von Neumann type and associated lattice equations are considered in Sec.IV. The next two sections are devoted to two- and multi-field generalizations of the von Neumann type equations. The examples presented include known lattice equations as well as some new ones. Particular cases of the Darboux transformation satisfying the conditions of the theorem of Sec.III are discussed in the Appendix.

\section{Lax pair and multi-field equations}

Let us begin with the overdetermined system of linear equations (Lax pair)

$$
\left\{\begin{array}{rl}
-i \dot{\psi} & =\psi A(\lambda) \\
z_{\lambda} \psi & =\psi H(\lambda)
\end{array} .\right.
$$

Here $\lambda$ and $z_{\lambda}$ are complex numbers, $\psi$ takes values in a given linear space $\mathcal{L}, A(\lambda)$ and $H(\lambda)$ are linear operators $\mathcal{L} \mapsto \mathcal{L}$ belonging to an associative ring, the dot denotes a derivative (i.e. an operator satisfying the Leibnitz rule). The compatibility condition of Eqs. (2) is

$$
i \dot{H}(\lambda)=[A(\lambda), H(\lambda)] .
$$


If we assume that the operators entering the Lax pair are rational functions of $\lambda$ with operator valued coefficients of the form

$$
\begin{aligned}
A(\lambda) & =\sum_{k=0}^{L} \lambda^{k} B_{k}+\sum_{k=1}^{M} \frac{1}{\lambda^{k}} C_{k}, \\
H(\lambda) & =\sum_{k=0}^{N} \lambda^{k} H_{k} .
\end{aligned}
$$

then Eq. (3) becomes equivalent to the following system of algebraic and differential relations between operators $B_{k}, C_{k}$ and $H_{k}$ :

$$
\begin{gathered}
\sum_{k=\max \{0, m-L\}}^{N}\left[B_{m-k}, H_{k}\right]=0 \quad(N<m \leq L+N), \\
\sum_{k=0}^{\min \{N, m+M\}}\left[C_{k-m}, H_{k}\right]=0 \quad(-M \leq m<0), \\
i \dot{H}_{m}=\sum_{k=\max \{0, m-L\}}^{m}\left[B_{m-k}, H_{k}\right]+\sum_{k=m+1}^{\min \{N, m+M\}}\left[C_{k-m}, H_{k}\right] \quad(0 \leq m \leq N) .
\end{gathered}
$$

The connection between operators $B_{k}, C_{k}$ and $H_{k}$, which is implied by Eqs. (6,7), is implicit. It is possible to express $B_{k}$ and $C_{k}$ explicitly in terms of the operators $H_{k}$ in order to satisfy Eqs. (6, (7) identically. Indeed, let us put

$$
\begin{aligned}
& B_{k}=\left.\frac{1}{(L-k) !}\left(\frac{d^{L-k}}{d \varsigma^{L-k}} f\left(\varsigma^{N} H\left(\varsigma^{-1}\right), \varsigma^{-1}\right)\right)\right|_{\varsigma=0}, \\
& C_{k}=\left.\frac{1}{(M-k) !}\left(\frac{d^{M-k}}{d \varepsilon^{M-k}} g(H(\varepsilon), \varepsilon)\right)\right|_{\varepsilon=0},
\end{aligned}
$$

where $f(X, \lambda)$ and $g(X, \lambda)$ are well defined functions of an operator $X$ and parameter $\lambda$. We assume hereafter that for any operator $X(\lambda)$, which is analytic in the neighborhood of the point $\lambda=\infty$, the function $f(X(\lambda), \lambda)$ is also analytic and the condition

$$
[f(X(\lambda), \lambda), X(\lambda)]=0 .
$$

is valid in this neighborhood as well. In the case of the function $g(X, \lambda)$, analogous properties have to take place in the neighborhood of the point $\lambda=0$. In particular we have

$$
[g(X(\lambda), \lambda), X(\lambda)]=0
$$

Eqs. (6,7) are fulfilled for any $B_{k}$ and $C_{k}$ defined by Eqs. (9,10) as a consequence of the following identities

$$
\begin{gathered}
\left.\frac{d^{N+L-m}}{d \varsigma^{N+L-m}}\left[f\left(\varsigma^{N} H\left(\varsigma^{-1}\right), \varsigma^{-1}\right), \varsigma^{N} H\left(\varsigma^{-1}\right)\right]\right|_{\varsigma=0} \equiv 0 \quad(N<m \leq L+N), \\
\left.\frac{d^{M+m}}{d \varepsilon^{M+m}}[g(H(\varepsilon), \varepsilon), H(\varepsilon)]\right|_{\varepsilon=0} \equiv 0 \quad(-M \leq m<0) .
\end{gathered}
$$

Using Eqs. (6,8) we can rewrite Eqs. (8) as

$$
-i \dot{H}_{m}=\sum_{k=m+1}^{N}\left[B_{m-k}, H_{k}\right]+\sum_{k=0}^{m}\left[C_{k-m}, H_{k}\right]
$$


where the coefficients $B_{k}$ and $C_{k}$ for $k<0$ are calculated accordingly to Eqs. (99,10). The operator $A(\lambda)$ given by $(4,9,9,10)$ is conveniently represented in the following equivalent way

$$
A(\lambda)=[F(H(\lambda), \lambda)]_{\infty}+[G(H(\lambda), \lambda)]_{0} .
$$

Here

$$
\begin{gathered}
F(H(\lambda), \lambda)=\lambda^{L} f\left(H(\lambda) / \lambda^{N}, \lambda\right), \\
G(H(\lambda), \lambda)=\lambda^{-M} g(H(\lambda), \lambda),
\end{gathered}
$$

symbols $[\ldots]_{\infty}$ and $[\ldots]_{0}$ denote the parts of the power expansions in $\lambda$ that contain nonnegative and negative powers respectively. These equations establish a connection between the coefficients of Lax pair (2).

In what follows we restrict our consideration to functions $f(X, \lambda)$ and $g(X, \lambda)$, which possess an additional property, namely they are covariant with respect to a similarity transformation $T$ :

$$
f\left(T^{-1} X T, \lambda\right)=T^{-1} f(X, \lambda) T, \quad g\left(T^{-1} X T, \lambda\right)=T^{-1} g(X, \lambda) T .
$$

Conditions (11, 12, 17) are not very restrictive and are satisfied, for example, by polynomials in $X$ and sums of negative powers of polynomials in $X$ with scalar coefficients. If $X$ is selfadjoint operator, then these conditions are valid for all $f(X, \lambda)$ and $g(X, \lambda)$ determined via the spectral theorem.

\section{Darboux covariance}

Let us consider the transformation

$$
\psi[1]=\psi D(\lambda)
$$

where ...[1] denotes the image under the transformation, and $D(\lambda)$ is an invertible linear operator depending on $\lambda$. We say that the Lax pair (2) is Darboux covariant with respect to transformation $\{\psi, A(\lambda), H(\lambda)\} \rightarrow\{\psi[1], A[1](\lambda), H[1](\lambda)\}$ if the following equations hold

$$
\left\{\begin{aligned}
-i \dot{\psi}[1] & =\psi[1] A[1](\lambda) \\
z_{\lambda} \psi[1] & =\psi[1] H[1](\lambda)
\end{aligned}\right.
$$

and the structure of $A[1](\lambda)$ and $H[1](\lambda)$ is the same as the structure of $A(\lambda)$ and $H(\lambda)$. The notion of "structure" means that the shapes of the coefficients of Lax pairs (2) and (19) are the same. The most important point is that the locations of singularities of $A(\lambda)$ and $A[1](\lambda), H(\lambda)$ and $H[1](\lambda)$ and their types should coincide. The transformations of the form (18,20,21) that satisfy these conditions are called Darboux transformations [13]. These transformations allow one to generate the hierarchies of solutions of nonlinear equations admitting the compatibility condition representation and of associated Lax pairs. In finite dimensional (matrix) cases $D(\lambda)$ is termed the Darboux matrix [14].

Substituting (18) into (19) we obtain expressions for the coefficients of the transformed Lax pair

$$
\begin{gathered}
A[1](\lambda)=-i D(\lambda)^{-1} \dot{D}(\lambda)+D(\lambda)^{-1} A(\lambda) D(\lambda) . \\
H[1](\lambda)=D(\lambda)^{-1} H(\lambda) D(\lambda) .
\end{gathered}
$$

If $D(\lambda)$ and $D(\lambda)^{-1}$ are regular on the plane of parameter $\lambda$ at singular points of the coefficients of Lax pair then the sufficient condition for $D(\lambda)$ to define the Darboux transformation 
comes from the requirement that the right hand sides in Eqs. (20,21) have no the singularities at the points, which are the singular point of $D(\lambda)$ and $D^{-1}(\lambda)$. We refer the Reader to the Appendix, where the examples of such transformation are presented.

The following theorem gives sufficient conditions of covariance of Eq. (14), which explicitly connects the coefficients of the Lax pair (2), with respect to the Darboux transformation $(18,21,21)$.

Theorem. If $D(\lambda)$ and $D(\lambda)^{-1}$ are rational functions in $\lambda$ that have poles at finite points $\mu_{1}, \ldots, \mu_{s}$ and $\mu_{s+1}, \ldots, \mu_{S}$ respectively, $\mu_{k} \neq 0(k=1, \ldots, S)$ and $\left[D(\lambda)^{-1} \dot{D}(\lambda)\right]_{\infty}=0$, then Eq. (14) is Darboux covariant.

Proof: Substituting (14) into (20) yields

$$
A[1](\lambda)=-i D(\lambda)^{-1} \dot{D}+D(\lambda)^{-1}[F(H(\lambda), \lambda)]_{\infty} D(\lambda)+D(\lambda)^{-1}[G(H(\lambda), \lambda)]_{0} D(\lambda) .
$$

It is enough to show that

$$
A[1](\lambda)=[F(H[1](\lambda), \lambda)]_{\infty}+[G(H[1](\lambda), \lambda)]_{0} .
$$

The right hand side of (22) is a rational function of $\lambda$ with poles at most at $\mu_{1}, \ldots, \mu_{S}$ and $0, \infty$. Therefore the following decomposition holds

$$
A[1](\lambda)=\sum_{k=1}^{S}[A[1](\lambda)]_{\mu_{k}}+[A[1](\lambda)]_{\infty}+[A[1](\lambda)]_{0}
$$

where $[A[1](\lambda)]_{\mu_{k}}$ is the principal part of the Laurent expansion of $A[1](\lambda)$ at the point $\lambda=\mu_{k}$. Since the first term in formula (22) can have poles at most at $\mu_{1}, \ldots, \mu_{S}$ and vanishes as $\lambda \rightarrow \infty$, we obtain

$$
\begin{aligned}
{[A[1](\lambda)]_{\infty} } & =\left[D(\lambda)^{-1}[F(H(\lambda), \lambda)]_{\infty} D(\lambda)\right]_{\infty}+\left[D(\lambda)^{-1}[G(H(\lambda), \lambda)]_{0} D(\lambda)\right]_{\infty} \\
{[A[1](\lambda)]_{0} } & =\left[D(\lambda)^{-1}[F(H(\lambda), \lambda)]_{\infty} D(\lambda)\right]_{0}+\left[D(\lambda)^{-1}[G(H(\lambda), \lambda)]_{0} D(\lambda)\right]_{0} .
\end{aligned}
$$

Using equalities

$$
\begin{gathered}
{\left[D(\lambda)^{-1}[F(H(\lambda), \lambda)]_{\infty} D(\lambda)\right]_{\infty}=\left[D(\lambda)^{-1} F(H(\lambda), \lambda) D(\lambda)\right]_{\infty},} \\
{\left[D(\lambda)^{-1}[F(H(\lambda), \lambda)]_{\infty} D(\lambda)\right]_{0}=\left[\left[D(\lambda)^{-1} F(H(\lambda), \lambda) D(\lambda)\right]_{\infty}\right]_{0}=0,} \\
{\left[D(\lambda)^{-1}[G(H(\lambda), \lambda)]_{0} D(\lambda)\right]_{\infty}=\left[\left[D(\lambda)^{-1} G(H(\lambda), \lambda) D(\lambda)\right]_{0}\right]_{\infty}=0,} \\
{\left[D(\lambda)^{-1}[G(H(\lambda), \lambda)]_{0} D(\lambda)\right]_{0}=\left[D(\lambda)^{-1} G(H(\lambda), \lambda) D(\lambda)\right]_{0} .}
\end{gathered}
$$

we have

$$
\begin{gathered}
{[A[1](\lambda)]_{\infty}=\left[D(\lambda)^{-1} F(H(\lambda), \lambda) D(\lambda)\right]_{\infty},} \\
{[A[1](\lambda)]_{0}=\left[D(\lambda)^{-1} G(H(\lambda), \lambda) D(\lambda)\right]_{0} .}
\end{gathered}
$$

It follows from the definition of the Darboux transformation that for $k=1, \ldots, S$

$$
[A[1](\lambda)]_{\mu_{k}} \equiv 0
$$

Combining (24 27) and taking into account (21), (15 17), we get (23). 
It is well known that the conditions (27) can be solved resulting in an explicit expression for $D(\lambda)$ in terms of solutions of the Lax pair (2) and a dual pair, which belong to the kernel of this operator or its inverse one. For this reason, we prefer to use the name of the Darboux transformation technique instead of the dressing method. In the Appendix we give examples of the Darboux transformations, which satisfy the conditions of our Theorem and can be used to construct the hierarchies of solutions of nonlinear equations (8) under constraints (9, 10). The applications of the Darboux transformation technique to certain nonlinear equations of von Neumann type, including an equation in infinite dimensional case, which are of interest in connection with quantum mechanics and statistical physics, can be found in [8, 9, 10].

If operator $T$ in Eqs. (17) is independent of $\lambda$, then the expressions in right hand sides of these formulas correspond to so-called gauge transformation of wave function

$$
\psi \rightarrow \tilde{\psi}=\psi T
$$

The case of the Lax pairs with $g(X, \lambda) \equiv 0$ is gauge equivalent to the case $f(X, \lambda) \equiv 0$ if $T$ solves equation

$$
i \dot{T}=B_{0} T \text {. }
$$

Some of the lattice equations presented in the next sections are the compatibility condition of gauge equivalent Lax pairs.

\section{Equations of von Neumann type (one-field equa- tions)}

In this section we consider the case $N=1$, i.e.,

$$
H(\lambda)=\lambda H_{1}+H_{0}
$$

The compatibility condition of the Lax pair gives us the equation

$$
\begin{gathered}
i \dot{H}_{0}=\left[B_{0}, H_{0}\right]+\left[C_{1}, H_{1}\right], \\
\dot{H}_{1}=0,
\end{gathered}
$$

where

$$
\begin{gathered}
\left.B_{0}=\frac{1}{L !}\left(\frac{d^{L}}{d \varsigma^{L}} f\left(H_{1}+\varsigma H_{0}\right), \varsigma^{-1}\right)\right)\left.\right|_{\varsigma=0}, \\
C_{1}=\left.\frac{1}{(M-1) !}\left(\frac{d^{M-1}}{d \varepsilon^{M-1}} g\left(\varepsilon H_{1}+H_{0}, \varepsilon\right)\right)\right|_{\varepsilon=0} .
\end{gathered}
$$

We refer to this case as "one-field" because $H_{1}$ is the constant operator. The right-hand side of Eq. (28) combines both types of the nonlinearities as in the equations of von Neumann type (11). The nonlinear equations corresponding to simplest choices of functions $f(X, \lambda)$ and $g(X, \lambda)$ are presented below. We also obtain the equations that follow them if the matrix coefficients $H_{1}$ and $H_{0}$ are defined in a special manner.

IV.1 $f(X, \lambda)=i X^{l}(l \in \mathbf{N}), g(X, \lambda)=0, L=1$

The compatibility condition leads to the equation

$$
\dot{H}_{0}=\sum_{m=1}^{l}\left[H_{1}^{m-1} H_{0} H_{1}^{l-m}, H_{0}\right] .
$$


This equation with $l=2$ is multi-dimensional Euler's top equation [15, 16, 17]. Darboux covariance of Eq. (31) and associated Lax pair was proved in 9].

Let matrices $H_{1}$ and $H_{0}$ have the form

$$
H_{1, k j}=\delta_{k, j-1}, H_{0, k j}=\rho_{k} \delta_{k, j+l-1}
$$

Then Eq. (31) yields

$$
\dot{\rho}_{k}=\rho_{k} \sum_{m=1}^{l-1}\left(\rho_{k+m}-\rho_{k-m}\right) .
$$

These equations are known as the Bogoyavlenskii lattice [18. In the case $l=2$ Eqs. (33) coincide with the Volterra system [19]

$$
\dot{\rho}_{k}=\rho_{k}\left(\rho_{k+1}-\rho_{k-1}\right),
$$

which describes stimulated scattering of plasma oscillations by ions [20].

IV.2 $f(X, \lambda)=i X^{-n}(n \in \mathbf{N}), g(X, \lambda)=0, L=1$

In this case Eq. (28) is written as given

$$
\dot{H}_{0}=-\sum_{m=1}^{n}\left[H_{1}^{-m} H_{0} H_{1}^{m-n-1}, H_{0}\right]
$$

The Bogoyavlenskii lattice (33) with $l=n+1$ follows this equation if matrices $H_{1}$ and $H_{0}$ are chosen in the form

$$
H_{1, k j}=\delta_{k, j-1}, H_{0, k j}=\rho_{k} \delta_{k, j-n-1} .
$$

IV.3 $f(X, \lambda)=0, g(X, \lambda)=i X^{l}(l \in \mathbf{N}), M=1$

From Eq. (28) we have

$$
\dot{H}_{0}=\left[H_{0}^{l}, H_{1}\right]
$$

Assuming that matrices $H_{1}$ and $H_{0}$ are represented in the following manner

$$
H_{1, k j}=\delta_{k, j-l+1}, H_{0, k j}=\rho_{k} \delta_{k, j+1}
$$

we obtain the well known lattice [18]

$$
\dot{\rho}_{k}=\prod_{m=0}^{l-1} \rho_{k-m}-\prod_{m=0}^{l-1} \rho_{k+m} .
$$

These equations with $l=2$ are obviously reduced to the Volterra system (34). If we put

$$
\rho_{k}=\exp \left(u_{k}\right)
$$

then Eqs. (39) read as

$$
\dot{u}_{k}=\exp \left(\sum_{m=1}^{l-1} u_{k-m}\right)-\exp \left(\sum_{m=1}^{l-1} u_{k+m}\right) .
$$

As it was noted at the end of Sec.III this case is gauge equivalent to the case IV.1. 
IV.4 $f(X, \lambda)=0, g(X, \lambda)=i X^{l}(l \in \mathbf{N}), M=2$

Eq. (28) yields

$$
\dot{H}_{0}=\sum_{m=0}^{l-1}\left[H_{0}^{m} H_{1} H_{0}^{l-m-1}, H_{1}\right]
$$

Supposing

$$
H_{1, k j}=\delta_{k, j-l+2}, H_{0, k j}=\rho_{k} \delta_{k, j+2},
$$

we have

$$
\dot{\rho}_{k}=\sum_{m=0}^{l-1}\left(\prod_{i=0}^{m-1} \rho_{k-2 i} \prod_{i=0}^{l-m-2} \rho_{k+2 i-l+2}-\prod_{i=0}^{m-1} \rho_{k-2 i+l-2} \prod_{i=0}^{l-m-2} \rho_{k+2 i}\right) .
$$

(It is assumed hereafter that $\prod_{i=0}^{m} \cdots i=1$ if $m<0$.) In the case $l=3$ these equations are equivalent to the Volterra system (34).

$$
\text { IV.5 } f(X, \lambda)=0, g(X, \lambda)=i X^{-l}(l \in \mathbf{N}), M=1
$$

Eq. (28) takes the form

$$
\dot{H}_{0}=\left[H_{0}^{-l}, H_{1}\right] .
$$

If matrices $H_{1}$ and $H_{0}$ are defined as follows

$$
H_{1, k j}=\delta_{k, j+l+1}, \quad H_{0, k j}=\rho_{k} \delta_{k, j+1},
$$

then the compatibility condition implies

$$
\dot{\rho}_{k}=\prod_{m=1}^{l} \rho_{k+m}^{-1}-\prod_{m=1}^{l} \rho_{k-m}^{-1} .
$$

Introducing two sets of new dependent variables

$$
\begin{gathered}
\rho_{k}=\exp \left(-u_{k}\right), \\
v_{k}=\rho_{k}^{-1},
\end{gathered}
$$

we obtain equivalent representations of Eqs. (46)

$$
\dot{u}_{k}=\exp \left(\sum_{m=0}^{l} u_{k-m}\right)-\exp \left(\sum_{m=0}^{l} u_{k+m}\right)
$$

and

$$
\dot{v}_{k}=v_{k}^{2}\left(\prod_{m=1}^{l} v_{k-m}-\prod_{m=1}^{l} v_{k+m}\right) .
$$

Eqs. (47) with $l=2$ were studied in [21. Lax pair for Eqs. (48) was found in 18]. These equations in the case $l=1$ obey a symmetry $v_{k} \rightarrow-v_{k}$ and look like a natural generalization of the Volterra system (34). 
IV.6 $f(X, \lambda)=0, g(X, \lambda)=i X^{-l}(l \in \mathbf{N}), M=2$

In this case Eq. (28) is written in the next manner

$$
\dot{H}_{0}=-\sum_{m=1}^{l}\left[H_{0}^{-m} H_{1} H_{0}^{m-l-1}, H_{1}\right] .
$$

If matrices $H_{1}$ and $H_{0}$ are defined as follows

$$
H_{1, k j}=\delta_{k, j+l+2}, \quad H_{0, k j}=\rho_{k} \delta_{k, j+2},
$$

then we come to equations

$$
\dot{\rho}_{k}=\sum_{m=1}^{l}\left(\prod_{i=1}^{m} \rho_{k+2 i-l-2}^{-1} \prod_{i=0}^{l-m} \rho_{k-2 i-2}^{-1}-\prod_{i=1}^{m} \rho_{k+2 i}^{-1} \prod_{i=0}^{l-m} \rho_{k-2 i+l}^{-1}\right) .
$$

IV.7 $f(X, \lambda)=0, g(X, \lambda)=g(X), M=1$

Here we have

$$
i \dot{H}_{0}=\left[g\left(H_{0}\right), H_{1}\right] .
$$

By construction, $g\left(H_{0}\right)$ commutes with $H_{0}$. The Lax representation and Darboux covariance properties of Eq. (52) with arbitrary well-defined function $g(X)$ were established in [10]. The cases $g(X)=i X^{3}$ and $g(X)=i X^{-1}$ were considered in [22] in the framework of the symmetry approach to the classification problem of integrable equations on free associative rings. The Lax representation for the equations in these cases seems to be new.

$$
\text { IV.8 } f(X, \lambda)=X^{4}, g(X, \lambda)=0, L=2
$$

The compatibility condition (28) becomes

$$
i \dot{H}_{0}=\left[h\left(H_{0}\right), H_{0}\right]=\left[H_{1}, F\left(H_{0}\right)\right]
$$

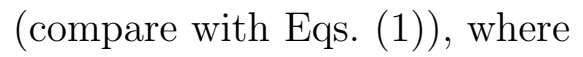

$$
\begin{gathered}
h\left(H_{0}\right)=H_{0}^{2} H_{1}^{2}+H_{0} H_{1} H_{0} H_{1}+H_{0} H_{1}^{2} H_{0}+H_{1} H_{0}^{2} H_{1}+H_{1} H_{0} H_{1} H_{0}+H_{1}^{2} H_{0}^{2}, \\
F\left(H_{0}\right)=H_{0}^{3} H_{1}+H_{0}^{2} H_{1} H_{0}+H_{0} H_{1} H_{0}^{2}+H_{1} H_{0}^{3} .
\end{gathered}
$$

Let us note that, contrary to the previous example, $\left[F\left(H_{0}\right), H_{0}\right] \neq 0$. We refer to the maps $H_{0} \mapsto F\left(H_{0}\right)$ of such a kind as nonabelian functions, or nonabelian nonlinearities [12]. This example is a particular case of the equations (41).

\section{Two-Field Equations}

A few examples of systems appearing if $N=2$ are considered in this section. 
V.1 $f(X, \lambda)=i X, g(X, \lambda)=0, L=1$

The compatibility condition (8) leads to equations

$$
\begin{aligned}
& \dot{H}_{0}=\left[H_{1}, H_{0}\right], \\
& \dot{H}_{1}=\left[H_{2}, H_{0}\right], \\
& \dot{H}_{2}=0 .
\end{aligned}
$$

It is checked immediately that functions

$$
\begin{aligned}
& F_{1}=\left(H_{2}-\sigma H_{0}\right) /(2 i), \\
& F_{2}=\left(H_{2}+\sigma H_{0}\right) / 2, \\
& F_{3}=H_{1} /(2 i),
\end{aligned}
$$

where $\sigma$ is a parameter $(\sigma \neq 0)$, satisfy equations

$$
\begin{aligned}
& \dot{F}_{1}=\left[F_{2}, F_{3}\right]+i\left[F_{3}, F_{1}\right], \\
& \dot{F}_{2}=\left[F_{3}, F_{1}\right]+i\left[F_{3}, F_{2}\right], \\
& \dot{F}_{3}=\sigma\left[F_{1}, F_{2}\right] .
\end{aligned}
$$

In terms of new dependent variables

$$
f_{k}=g F_{k} g^{-1} \quad(k=1,2,3),
$$

where $g$ solves equation

$$
\dot{g}=-i g F_{3}
$$

Eqs. (54 56) are rewritten as

$$
\begin{aligned}
& \dot{f}_{1}=\left[f_{2}, f_{3}\right], \\
& \dot{f}_{2}=\left[f_{3}, f_{1}\right], \\
& \dot{\dot{f}_{3}}=\sigma\left[f_{1}, f_{2}\right] .
\end{aligned}
$$

If we impose condition $f_{k}^{+}=-f_{k}$, then $\sigma$ has to be real. This system with $\sigma=1$ is known as Nahm equations [23, 24]. It will be shown in the next subsection that this case is also connected with Toda lattice equation.

$$
\text { V.2 } f(X, \lambda)=i X^{l}(l \in \mathbf{N}), g(X, \lambda)=0, L=1
$$

From Eqs. (8) we have

$$
\begin{aligned}
& \dot{H}_{0}=\sum_{m=1}^{l}\left[H_{2}^{m-1} H_{1} H_{2}^{l-m}, H_{0}\right], \\
& \dot{H}_{1}=\left[H_{2}^{l}, H_{0}\right]+\sum_{m=1}^{l}\left[H_{2}^{m-1} H_{1} H_{2}^{l-m}, H_{1}\right], \\
& \dot{H}_{2}=0 .
\end{aligned}
$$

If we put

$$
H_{2, k j}=\delta_{k, j-1}, \quad H_{1, k j}=h_{k} \delta_{k, j+l-1}, \quad H_{0, k j}=\rho_{k} \delta_{k, j+2 l-1},
$$


then Eqs. (57,58) read as

$$
\left\{\begin{array}{l}
\dot{h}_{k}=\rho_{k+l}-\rho_{k}+h_{k} \sum_{m=1}^{l-1}\left(h_{k+m}-h_{k-m}\right), \\
\dot{\rho}_{k}=\rho_{k} \sum_{m=0}^{l-1}\left(h_{k+m}-h_{k-l-m}\right) .
\end{array}\right.
$$

Let

$$
h_{k}=\dot{\sigma}_{k}
$$

If coefficients $\rho_{k}$ are chosen as given

$$
\rho_{k}=C \mathrm{e}^{\sum_{m=0}^{l-1}\left(\sigma_{k+m}-\sigma_{k-l-m}\right)}
$$

( $C$ is arbitrary constant), then system (60) is equivalent to the following equations

$$
\ddot{\sigma}_{k}=C\left(\mathrm{e}^{\sum_{m=0}^{l-1}\left(\sigma_{k+m+l}-\sigma_{k-m}\right)}-\mathrm{e}^{\sum_{m=0}^{l-1}\left(\sigma_{k+m}-\sigma_{k-l-m}\right)}\right)+\dot{\sigma}_{k} \sum_{m=1}^{l-1}\left(\dot{\sigma}_{k+m}-\dot{\sigma}_{k-m}\right) .
$$

Assuming $l=1$ and $C=1$ we come to the Toda lattice equation [25, 26, 27]

$$
\ddot{\sigma}_{k}=\mathrm{e}^{\sigma_{k+1}}-\sigma_{k}-\mathrm{e}^{\sigma_{k}-\sigma_{k-1}}
$$

Eqs. (61) can be viewed as a generalization of the Toda lattice on the systems of particles interacting with finite number of nearest neighborhoods. For $n \times n$ matrices, these equations admit additional reductions

$$
\begin{gathered}
\sigma_{m+1+k}=-\sigma_{m+1-k} \quad \text { if } \quad n=2 m+1, \\
\sigma_{m+k}=-\sigma_{m+1-k} \quad \text { if } \quad n=2 m
\end{gathered}
$$

or

$$
\sigma_{m+k}=-\sigma_{m-k} \quad \text { if } \quad n=2 m
$$

$(k=0, \ldots, m)$. In the case $l=1$, these reductions lead to generalized periodic Toda lattices, whose connection with the root systems of semisimple Lie algebras was established in [28.

$$
\text { V.3 } f(X, \lambda)=i X^{-l}(l \in \mathbf{N}), g(X, \lambda)=0, L=1
$$

The compatibility condition in this case yields

$$
\begin{aligned}
& \dot{H}_{0}=-\sum_{m=1}^{l}\left[H_{2}^{-m} H_{1} H_{2}^{m-l-1}, H_{0}\right], \\
& \dot{H}_{1}=\left[H_{2}^{-l}, H_{0}\right]-\sum_{m=1}^{l}\left[H_{2}^{-m} H_{1} H_{2}^{m-l-1}, H_{1}\right] \\
& \dot{H}_{2}=0 .
\end{aligned}
$$

If $H_{2}, H_{1}$ and $H_{0}$ are defined in the following manner

$$
H_{2, k j}=\delta_{k, j+1}, \quad H_{1, k j}=h_{k} \delta_{k, j+l+1}, \quad H_{0, k j}=\rho_{k} \delta_{k, j+2 l+1},
$$


then Eqs. (63,64) are written as

$$
\left\{\begin{array}{l}
\dot{h}_{k}=\rho_{k+l}-\rho_{k}-h_{k} \sum_{m=1}^{l}\left(h_{k+m}-h_{k-m}\right), \\
\dot{\rho}_{k}=\rho_{k} \sum_{m=1}^{l}\left(h_{k-l-m}-h_{k+m}\right) .
\end{array}\right.
$$

In the case $l=1$ these equations are so-called Belov-Chaltikian lattice [29]. The bilinear approach was applied to Belov-Chaltikian lattice in 30.

Expressing dependent variables in the terms of new ones

$$
\begin{gathered}
h_{k}=\dot{\sigma}_{k}, \\
\rho_{k}=C \mathrm{e}^{\sum_{m=1}^{l}\left(\sigma_{k-l-m}-\sigma_{k+m}\right)}
\end{gathered}
$$

( $C$ is a constant) we reduce Eqs. (66) to the Toda-type lattice equations

$$
\ddot{\sigma}_{k}=C\left(\mathrm{e}^{\sum_{m=1}^{l}\left(\sigma_{k-m}-\sigma_{k+l+m}\right)}-\mathrm{e}^{\sum_{m=1}^{l}\left(\sigma_{k-l-m}-\sigma_{k+m}\right)}\right)-\dot{\sigma}_{k} \sum_{m=1}^{l}\left(\dot{\sigma}_{k+m}-\dot{\sigma}_{k-m}\right) .
$$

$\mathbf{V . 4} f(X, \lambda)=0, g(X, \lambda)=i X^{l}(l \in \mathbf{N}), M=1$

In this case Eqs. (8) are rewritten as given

$$
\begin{aligned}
\dot{H}_{0} & =\left[H_{0}^{l}, H_{1}\right], \\
\dot{H}_{1} & =\left[H_{0}^{l}, H_{2}\right], \\
\dot{H}_{2} & =0 .
\end{aligned}
$$

If matrices $H_{2}, H_{1}$ and $H_{0}$ have the form

$$
H_{2, k j}=\delta_{k, j-2 l+1}, \quad H_{1, k j}=h_{k} \delta_{k, j-l+1}, \quad H_{0, k j}=\rho_{k} \delta_{k, j+1},
$$

then Eqs. (68,69) yield

$$
\left\{\begin{array}{l}
\dot{h}_{k}=\prod_{m=0}^{l-1} \rho_{k-m}-\prod_{m=0}^{l-1} \rho_{k+l+m} \\
\dot{\rho}_{k}=h_{k-l} \prod_{m=0}^{l-1} \rho_{k-m}-h_{k} \prod_{m=0}^{l-1} \rho_{k+m}
\end{array}\right.
$$

These equations with $l=1$ are equivalent to the Toda lattice (62).

$\mathbf{V . 5} f(X, \lambda)=0, g(X, \lambda)=i X^{l}(l \in \mathbf{N}), M=2$

From Eqs. (8) we have

$$
\begin{aligned}
\dot{H}_{0} & =\left[H_{0}^{l}, H_{2}\right]+\sum_{m=0}^{l-1}\left[H_{0}^{m} H_{1} H_{0}^{l-m-1}, H_{1}\right] \\
\dot{H}_{1} & =\sum_{m=0}^{l-1}\left[H_{0}^{m} H_{1} H_{0}^{l-m-1}, H_{2}\right] \\
\dot{H}_{2} & =0
\end{aligned}
$$


Taking matrices $H_{2}, H_{1}$ and $H_{0}$ as given

$$
H_{2, k j}=\delta_{k, j-2 l+2}, \quad H_{1, k j}=h_{k} \delta_{k, j-l+2}, \quad H_{0, k j}=\rho_{k} \delta_{k, j+2},
$$

we put compatibility condition into the form

$$
\left\{\begin{array}{l}
\dot{h}_{k}=\sum_{m=0}^{l-1}\left(h_{k-2 m} \prod_{i=0}^{m-1} \rho_{k-2 i} \prod_{i=0}^{l-m-2} \rho_{k+2 i-l+2}-h_{k-2 m+l-2} \prod_{i=0}^{m-1} \rho_{k-2 i+l-2} \prod_{i=0}^{l-m-2} \rho_{k+2 i}\right), \\
\dot{\rho}_{k}=h_{k-l} \sum_{m=0}^{l-1} h_{k-2 m} \prod_{i=0}^{m-1} \rho_{k-2 i} \prod_{i=0}^{l-m-2} \rho_{k+2 i-l+2}- \\
-h_{k} \sum_{m=0}^{l-1} h_{k-2 m-2} \prod_{i=0}^{m-1} \rho_{k-2 i-2} \prod_{i=0}^{l-m-2} \rho_{k+2 i-l}+\prod_{i=0}^{l-1} \rho_{k-2 i}-\prod_{i=0}^{l-1} \rho_{k+2 i} .
\end{array}\right.
$$

In the case $l=2$ and $h_{k}=0$ these equations coincide with the Volterra system (34).

V.6 $f(X, \lambda)=0, g(X, \lambda)=i X^{-l}(l \in \mathbf{N}), M=1$

In this case Eqs. (8) yield

$$
\begin{aligned}
\dot{H}_{0} & =\left[H_{0}^{-l}, H_{1}\right], \\
\dot{H}_{1} & =\left[H_{0}^{-l}, H_{2}\right], \\
\dot{H}_{2} & =0 .
\end{aligned}
$$

Let the matrices $H_{2}, H_{1}$ and $H_{0}$ be represented in the following manner

$$
H_{2, k j}=\delta_{k, j+2 l+1}, \quad H_{1, k j}=h_{k} \delta_{k, j+l+1}, \quad H_{0, k j}=\rho_{k} \delta_{k, j+1} .
$$

The compatibility condition leads to the system

$$
\left\{\begin{array}{l}
\dot{h}_{k}=\prod_{m=1}^{l} \rho_{k+m}^{-l}-\prod_{m=1}^{l} \rho_{k-l-m}^{-l}, \\
\dot{\rho}_{k}=h_{k+l} \prod_{m=1}^{l} \rho_{k+m}^{-l}-h_{k} \prod_{m=1}^{l} \rho_{k-m}^{-l} .
\end{array}\right.
$$

$$
\text { V.7 } f(X, \lambda)=0, g(X, \lambda)=i X^{-l}(l \in \mathbf{N}), M=2
$$

Eqs. (8) give

$$
\begin{aligned}
& \dot{H}_{0}=\left[H_{0}^{-l}, H_{2}\right]-\sum_{m=1}^{l}\left[H_{0}^{-m} H_{1} H_{0}^{m-l-1}, H_{1}\right], \\
& \dot{H}_{1}=-\sum_{m=1}^{l}\left[H_{0}^{-m} H_{1} H_{0}^{m-l-1}, H_{2}\right], \\
& \dot{H}_{2}=0 .
\end{aligned}
$$

Supposing

$$
H_{2, k j}=\delta_{k, j+2 l+2}, \quad H_{1, k j}=h_{k} \delta_{k, j+l+2}, \quad H_{0, k j}=\rho_{k} \delta_{k, j+2},
$$


we have

$$
\left\{\begin{array}{l}
\dot{h}_{k}=\sum_{m=1}^{l}\left(h_{k+2 m-2 l-2} \prod_{i=1}^{m} \rho_{k+2 i-2 l-2}^{-1} \prod_{i=0}^{l-m} \rho_{k-2 i-l-2}^{-1}-h_{k+2 m} \prod_{i=1}^{m} \rho_{k+2 i}^{-1} \prod_{i=0}^{l-m} \rho_{k-2 i+l}^{-1}\right), \\
\dot{\rho}_{k}=h_{k} \sum_{m=1}^{l} h_{k+2 m-l-2} \prod_{i=1}^{m} \rho_{k+2 i-l-2}^{-1} \prod_{i=0}^{l-m} \rho_{k-2 i-2}^{-1}- \\
-h_{k+l} \sum_{m=1}^{l} h_{k+2 m} \prod_{i=1}^{m} \rho_{k+2 i}^{-1} \prod_{i=0}^{l-m} \rho_{k-2 i+l}^{-1}+\prod_{i=1}^{l} \rho_{k+2 i}^{-1}-\prod_{i=1}^{l} \rho_{k-2 i}^{-1} .
\end{array}\right.
$$

\section{V.8 $f(X, \lambda)=i X^{2}, g(X, \lambda)=0, L=2$}

The compatibility condition (8) in this case is written as

$$
\begin{aligned}
\dot{H}_{0} & =\left[H_{1}^{2}, H_{0}\right]+\left[H_{2}, H_{0}^{2}\right], \\
\dot{H}_{1} & =\left[H_{2}, H_{0} H_{1}+H_{1} H_{0}\right], \\
\dot{H}_{2} & =0 .
\end{aligned}
$$

Taking matrices $H_{2}, H_{1}$ and $H_{0}$ as follows

$$
H_{2, k j}=\delta_{k, j-1}, \quad H_{1, k j}=h_{k} \delta_{k, j}, \quad H_{0, k j}=\rho_{k} \delta_{k, j+1},
$$

we obtain

$$
\left\{\begin{array}{l}
\dot{h}_{k}=\rho_{k+1}\left(h_{k+1}+h_{k}\right)-\rho_{k}\left(h_{k}+h_{k-1}\right), \\
\dot{\rho}_{k}=\rho_{k}\left(\rho_{k+1}-\rho_{k-1}+h_{k}^{2}-h_{k-1}^{2}\right) .
\end{array}\right.
$$

This system is the first member of the hierarchy of higher Toda lattices [31, 32]. In the case $h_{k}=0$ this system is nothing but the Volterra system (34).

\section{Multi-Field Equations}

In this section we present nonlinear equations that follow Eq. (8) with positive integer $N$ for special choices of functions $f(X, \lambda)$ and $g(X, \lambda)$.

$$
\text { VI.1 } f(X, \lambda)=i X^{l}(l \in \mathbf{N}), g(X, \lambda)=0, L=1
$$

The compatibility condition is

$$
\dot{H}_{i}=\left[H_{N}^{l}, H_{i-1}\right]+\sum_{m=1}^{l}\left[H_{N}^{m-1} H_{N-1} N_{N}^{l-m}, H_{i}\right]
$$

$(i=0, \ldots, N)$. If we put

$$
H_{i, k j}=\rho_{k}^{(i)} \delta_{k, j+(N-i) l-1}
$$

$\left(\rho_{k}^{(N)}=1\right)$, then Eqs. (86) give

$$
\dot{\rho}_{k}^{(i)}=\rho_{k+l}^{(i-1)}-\rho_{k}^{(i-1)}+\rho_{k}^{(i)} \sum_{m=0}^{l-1}\left(\rho_{k+m}^{(N-1)}-\rho_{k+(i-N+1) l-m}^{(N-1)}\right) .
$$


VI.2 $f(X, \lambda)=i X^{-l}(l \in \mathbf{N}), g(X, \lambda)=0, L=1$

In this case Eqs. (8) are written in the following way

$$
\dot{H}_{i}=\left[H_{N}^{-l}, H_{i-1}\right]-\sum_{m=1}^{l}\left[H_{N}^{-m} H_{N-1} N_{N}^{m-l-1}, H_{i}\right]
$$

$(i=0, \ldots, N)$. Assuming

$$
H_{i, k j}=\rho_{k}^{(i)} \delta_{k, j-(N-i) l-1}, \quad \rho_{k}^{(N)}=1,
$$

we obtain

$$
\dot{\rho}_{k}^{(i)}=\rho_{k-l}^{(i-1)}-\rho_{k}^{(i-1)}+\rho_{k}^{(i)} \sum_{m=1}^{l}\left(\rho_{k+(N-i-1) l+m}^{(N-1)}-\rho_{k-m}^{(N-1)}\right) .
$$

VI.3 $f(X, \lambda)=0, g(X, \lambda)=i X^{l}(l \in \mathbf{N}), M=1$

The compatibility condition (8) yield

$$
\dot{H}_{i}=\left[H_{0}^{l}, H_{i+1}\right]
$$

$(i=0, \ldots, N)$. Let matrices $H_{i}$ have the form

$$
H_{i, k j}=\rho_{k}^{(i)} \delta_{k, j-i l+1},
$$

where $\rho_{k}^{(N)}=1$. In this case Eqs. (92) lead to the following lattice equations

$$
\dot{\rho}_{k}^{(i)}=\rho_{k-l}^{(i+1)} \prod_{m=0}^{l-1} \rho_{k-m}^{(0)}-\rho_{k}^{(i+1)} \prod_{m=0}^{l-1} \rho_{k+i l+m}^{(0)} .
$$

The case $l=1$ was studied in [33, 34]

VI.4 $f(X, \lambda)=0, g(X, \lambda)=i X^{-l}(l \in \mathbf{N}), M=1$

From Eqs. (8) we have

$$
\dot{H}_{i}=\left[H_{0}^{-l}, H_{i+1}\right]
$$

$(i=0, \ldots, N)$. If matrices $H_{i}$ are defined as follows

$$
H_{i, k j}=\rho_{k}^{(i)} \delta_{k, j+i l+1},
$$

then Eqs. (95) give

$$
\dot{\rho}_{k}^{(i)}=\rho_{k+l}^{(i+1)} \prod_{m=1}^{l} \rho_{k+m}^{-1}-\rho_{k}^{(i+1)} \prod_{m=1}^{l} \rho_{k-i l-m}^{-1},
$$

where we use the notation

$$
\rho_{k}^{(0)}=\rho_{k}
$$

\section{Conclusion}

In future we will continue the study of the von Neumann type equations and their generalizations presented in the previous sections. The integrals and the multi-soliton solutions will be considered. An investigation of the hierarchies of symmetries and compatible flows for these equations can lead to new hierarchies of integrable equations [22, 35, 36, 37]. The results will be of special interest in the case of integrable lattice equations. It should be also mentioned that discretizations of the lattice equations attract large attention in recent years (see, e.g., 38 and references therein). 


\section{Acknowledgements}

N. V. U. is grateful to Jan L. Cieśliński and Marek Czachor for hospitality. This research was supported in part by the KBN grant 2 P03B 12622 (J. L. C.), the KBN grant 5 P03B 04020 (M. C.), Alexander von Humboldt foundation (M. C.) and Nokia-Poland (N. V. U.).

\section{Appendix. Darboux Transformations}

Here we discuss briefly some particular cases of the Darboux transformation, which satisfy the conditions of Theorem in Sec.II. Let the operator $D(\lambda)$ in (18) be represented as given

$$
D(\lambda)=\mathbf{1}+\frac{\nu-\mu}{\mu-\lambda} P
$$

where

$$
P=\frac{\varphi \chi}{(\chi, \varphi)}
$$

$\chi$ is a solution of the Lax pair (2) with parameter $\nu$ :

$$
\left\{\begin{aligned}
-i \dot{\chi} & =\chi A(\nu) \\
z_{\nu} \chi & =\chi H(\nu)
\end{aligned}\right.
$$

$\varphi$ is a solution of the dual Lax pair with parameter $\mu$ :

$$
\left\{\begin{aligned}
i \dot{\varphi} & =A(\mu) \varphi \\
z_{\mu} \varphi & =H(\mu) \varphi
\end{aligned}\right.
$$

$(\chi, \varphi)$ is a scalar product. It is obvious that $P^{2}=P$ and

$$
-i \dot{P}=P A(\nu) P_{\perp}-P_{\perp} A(\mu) P
$$

$\left(P_{\perp}=1-P\right)$. If coefficients of the transformed Lax pair (19) are defined by

$$
\begin{aligned}
& A(\lambda)[1]=\sum_{k=0}^{L} \lambda^{k} B_{k}[1]+\sum_{k=1}^{M} \frac{1}{\lambda^{k}} C_{k}[1], \\
& H(\lambda)[1]=\sum_{k=0}^{N} \lambda^{k} H_{k}[1]
\end{aligned}
$$

where

$$
\begin{aligned}
& B_{k}[1]=B_{k}+(\mu-\nu) \sum_{m=k+1}^{L}\left(\mu^{m-k-1} P_{\perp} B_{m} P-\nu^{m-k-1} P B_{m} P_{\perp}\right) \\
& C_{k}[1]=C_{k}-(\mu-\nu) \sum_{m=k}^{M}\left(\mu^{k-m-1} P_{\perp} C_{m} P-\nu^{k-m-1} P C_{m} P_{\perp}\right) \\
& H_{k}[1]=H_{k}+(\mu-\nu) \sum_{m=k+1}^{N}\left(\mu^{m-k-1} P_{\perp} H_{m} P-\nu^{m-k-1} P H_{m} P_{\perp}\right)
\end{aligned}
$$

then Eqs. (19) are identically fulfilled. This statement can be proved by direct computation. Since

$$
D(\lambda)^{-1}=1+\frac{\mu-\nu}{\nu-\lambda} P
$$


operators $D(\lambda)$ and $D(\lambda)^{-1}$ have poles in points $\mu$ and $\nu$. It is seen from Eq. (99) that Eq. (27) are valid.

The formulas written above form the so-called binary Darboux transformation. The corresponding Darboux transformation for an $n$-dimensional matrix case is produced from them if we assume in (98) that

$$
P=\varphi(\chi \varphi)^{-1} \chi
$$

where $\chi$ and $\varphi$ are respectively $m \times n$ and $n \times m$ matrix solutions of direct and dual Lax pairs. Some examples of Darboux transformations in infinite dimensional case, which are suitable for integrable lattice equations, were given in [13]. Very recently a new construction of the Darboux transformation in terms of Clifford numbers was described in [39].

\section{References}

[1] I.M. Gelfand, L.A. Dikii, Funct. Anal. Appl. 10, 259 (1976).

[2] B.A. Kupershmidt, Commun. Math. Phys. 99, 51 (1988).

[3] V.G. Drinfeld, V.V. Sokolov, J. Sov. Math. 30, 1975 (1985).

[4] M. Sato, Soliton Equations as Dynamical Systems on Infinite Grassmann Manifold, RIMS Kokyuroku, Kyoto Univ. 439, 30 (1981); M. Sato, Y. Sato, Soliton Equations as Dynamical Systems on Infinite Grassmann Manifold, in: Nonlinear Partial Differential Equations in Applied Science, Eds. H. Fujita, P.D. Lax, G. Strang (Kinokuniya/NorthHolland, Tokio, 1983) 259.

[5] Y. Cheng, J. Math. Phys. 33, 3774 (1992).

[6] J. Sidorenko, W. Strampp, J. Math. Phys. 34, 1429 (1993).

[7] M. Błaszak, Multi-Hamiltonian Theory of Dynamical Systems, (Springer-Verlag, Berlin-Heidelberg, 1998).

[8] S.B. Leble, M. Czachor, Phys. Rev. 58E, 7091 (1998).

[9] M. Kuna, M. Czachor, S.B. Leble, Phys. Lett. A255, 42 (1999).

[10] N.V. Ustinov, M. Czachor, M. Kuna, S.B. Leble, Phys. Lett. A279, 333 (2001).

[11] J.L. Cieśliński, How to Construct Darboux-Invariant Equations of von Neumann Type, in: Probing the Structure of Quantum Mechanics: Nonlinearity, Nonlocality, Computation, Axiomatics, Eds. D. Aerts, M. Czachor, T. Durt (World Scientific, Singapore, 2002) 324-334.

[12] N.V. Ustinov, M. Czachor, Darboux-Integrable Equations with Non-Abelian Nonlinearities, in: Probing the Structure of Quantum Mechanics: Nonlinearity, Nonlocality, Computation, Axiomatics, Eds. D. Aerts, M. Czachor, T. Durt (World Scientific, Singapore, 2002) 335-353.

[13] V.B. Matveev, M.A. Salle, Darboux Transformations and Solitons (Springer-Verlag, Berlin-Heidelberg, 1991).

[14] J. Cieśliński, J. Math. Phys. 36, 5670 (1995). 
[15] V.I. Arnold, Mathematical Methods of Classical Mechanics, (Springer-Verlag, BerlinHeidelberg, 1989).

[16] A.S. Mishchenko, Funct. Anal. Appl. 4, 232 (1970).

[17] S.V. Manakov, Funct. Anal. Appl. 10, 328 (1976).

[18] O.I. Bogoyavlenskii, Usp. Math. Nauk 46, 3 (1991); Russ. Math. Surv. 46, 1 (1991).

[19] V. Volterra, Leçons sur la Théorie Mathématique de la Lutte sur la Vie, (GautierVillars, Paris, 1931).

[20] V.E. Zakharov, S.L. Musher, A.M. Rubenchik, Pis'ma v ZETF 19, 249 (1974) (Engl. transl. JETP Lett. 19, 151 (1974)); Zh. Eksp. Theor. Fiz. 69, 155 (1975) (Engl. transl. Sov. Phys.-JETP 42, 80 (1976)).

[21] X.B. Hu, Y.T. Wu, Phys. Lett. A246, 523 (1998).

[22] A.V. Mikhailov, V.V. Sokolov, Theor. Math. Phys. 122, 72 (2000).

[23] W. Nahm, Phys. Lett. B90, 413 (1980).

[24] N.J. Hitchin, Commun. Math. Phys. 89, 145 (1983).

[25] M. Toda, J. Phys. Soc. Jpn. 22, 431 (1967).

[26] S.V. Manakov, Zh. Eksp. Theor. Fiz. 67, 543 (1974) (Engl. transl. Sov. Phys.-JETP 40, 269 (1975)).

[27] H. Flashka, Progr. Theor. Phys. 51, 703 (1974).

[28] O.I. Bogoyavlenskii, Commun. Math. Phys. 51, 201 (1976).

[29] A.A. Belov, K.D. Chaltikian, Phys. Lett. B309, 268 (1993).

[30] X.B. Hu, H.W. Tam, Glasgow Math. J. A43, 43 (2001).

[31] M. Toda, Theory of Nonlinear Lattice, (Springer-Verlag, New York, 1981).

[32] A.M. Perelomov, Integrable Systems of Classical Mechanics and Lie Algebras, (Nauka, Moskow, 1990).

[33] M. Błaszak, K. Marciniak, J. Math. Phys. 35, 4661 (1994).

[34] X.B. Hu, H.W. Tam, Rep. Math. Phys. 46, 99 (2000).

[35] A.K. Svinin, J. Phys. A: Math. \& Gen. 34, 10559 (2001); Theor. Math. Phys. 130, 15 (2002).

[36] V.E. Adler, A.B. Shabat, Theor. Math. Phys. 111, 323 (1997).

[37] V.E. Adler, V.G. Marihin, A.B. Shabat, Theor. Math. Phys. 129, 163 (2001).

[38] D. Levi, L. Martina, J. Phys. A: Math. \& Gen. 34, 10357 (2001).

[39] J.L. Cieśliński, J. Phys. A: Math. \& Gen. 33, L363 (2000); W. Biernacki, J.L. Cieśliński, Phys. Lett. A 288, 167 (2001). 